\title{
Understanding the effects of task-specific practice in the brain: Insights from individual-differences analyses
}

\author{
GIORGIO GANIS \\ Harvard University, Cambridge, Massachusetts \\ Massachusetts General Hospital Martinos Center, Charlestown, Massachusetts \\ and Harvard Medical School, Boston, Massachusetts \\ WILLIAM L. THOMPSON \\ Harvard University, Cambridge, Massachusetts \\ and \\ STEPHEN M. KOSSLYN \\ Harvard University, Cambridge, Massachusetts \\ and Massachusetts General Hospital, Boston, Massachusetts
}

\begin{abstract}
We used functional magnetic resonance imaging to study practice effects in different mental imagery tasks. The study was designed to address three general questions: First, are the results of standard group-based analyses the same as those of a regression method in which brain activation changes over individual participants are used to predict task performance changes? With respect to the effects of practice, the answer was clear: Group-based analyses produced different results from regression-based individual-differences analyses. Second, are all brain areas that predict practice effects consistently activated across participants? Again, the answer was clear: Most areas that predicted the effects of practice on performance were not activated consistently over participants. Finally, does practice affect different areas in different ways for different people in different tasks? The answer was again clear: The areas that predicted changes in performance with practice varied for the different tasks, but this was more dramatically and clearly revealed by the individual-differences analyses. In short, individual-differences analyses provided insights into the relation between changes in brain activation and changes in accompanying performance, and these insights were not provided by standard group-based analyses.
\end{abstract}

Neuroimaging has revealed that even apparently simple perceptual and cognitive tasks are carried out by numerous interconnected brain areas and that different tasks typically rely on partially overlapping sets of brain areas (e.g., Kandel \& Squire, 2000; Kosslyn, Thompson, \& Alpert, 1997; Smith \& Jonides, 1997). Research has also revealed that the more similar the tasks, the more brain areas are activated in common; for example, when participants performed different judgments with different stimuli in imagery and perception tasks, about two thirds of the same areas were activated in common (Kosslyn et al., 1997), whereas when participants performed the same judgment on imagined and visually perceived versions of the same stimuli, over $90 \%$ of the same brain areas were activated

This work was supported in part by Grants R01 MH060734-05A1 and R21 MH068610A-01A1 from the National Institute of Mental Health, Grant NMA201-01-C-0032 from the National Mapping Agency, and ROLE Grants REC-0106760 and REC-0411725 from the National Science Foundation. Correspondence concerning this article should be addressed to G. Ganis, Department of Psychology, Harvard University, 33 Kirkland St., Cambridge, MA 02138 (e-mail: ganis@wjh.harvard.edu).
(Ganis, Thompson, \& Kosslyn, 2004). The pattern of commonalities and differences between brain regions active in different tasks provides a plausible explanation for the pattern of impairments exhibited by neurological patients. For instance, consistent with the overlap between the neural activation elicited by visual imagery and visual perception, damage to the ventral temporal cortex often leads to parallel impairments in visual imagery and visual perception (Ganis, Thompson, Mast, \& Kosslyn, 2003). Conversely, the lack of common activation in some brain regions can explain why brain damage sometimes results in a dissociation between impairments in visual imagery and visual perception (Ganis et al., 2003).

Understanding which brain areas are recruited during specific tasks is important not only if we are to understand the effects of brain damage, but also if we are to understand many fundamental aspects of the relation between mind and brain - such as how drugs selectively affect performance and how genes affect cognition and affect. However, the neuroimaging findings that document the networks of areas activated while participants perform specific tasks rely on a number of key assumptions, 
and these assumptions may, at least sometimes, lead us to mischaracterize the pattern of brain activation that underlies task performance. In the present article, we will consider implications of three general methodological assumptions that characterize most neuroimaging studies.

First, most neuroimaging studies employ a subtraction logic, which entails comparing results from test and baseline (control) tasks. This logic is not new: It was originally developed over 100 years ago to study response times (RTs; Donders, 1868/1969) and has been used extensively in mental chronometry studies to infer the duration of mental processes (Posner, 1978). The primary assumptions underlying the subtractive logic are that (1) each task draws on a set of processes, (2) the processes are independent and additive, with each one carrying out a distinct mental operation (see Sternberg, 1969), and (3) it is possible to devise a control task that shares all processes but one with a test task (Sartori \& Umiltà, 2000). If these assumptions are met, subtracting activation in the simpler (baseline) task from that in the comparison (test) task can isolate the effects of specific individual processes. Violations of these assumptions can lead to artifactual results; for example, if a baseline task engages processes not engaged by the test task, the results of the subtraction will be contaminated by such baseline processes (Sartori \& Umiltà, 2000). Unfortunately, for the vast majority of tasks and cognitive processes, it is difficult to know the extent to which the additivity assumption holds. In the present study, we asked whether the results of subtraction are the same as those obtained with another method, where variations in activation are used to predict variations in performance of the task.

The second shared characteristic of most neuroimaging studies is that the data analyses seek to identify brain regions that are consistently activated across individuals in a particular task (relative to a control condition). This type of analysis rests on the assumption that all individuals perform a task in approximately the same way, engaging the same set of brain regions in a similar manner. Although there is reason to believe that this assumption is valid in some cases, especially for simple tasks, it is also true that individual differences in performance have been documented in many cognitive domains (e.g., Ackerman, Kyllonen, \& Roberts, 1999). In fact, brain regions that are not activated consistently across individuals may, nonetheless, be key contributors to the performance of interest - and these brain regions are unlikely to be detected in a standard group average analysis because, by definition, they do not generalize across individuals (and the error terms in the analyses rely on such generalization). To examine this assumption, we compared the results of group-based analyses with those of regression-based individual-differences analyses. The latter analyses rely not simply on documenting differences in activation over participants, but rather, on showing that these differences predict performance (and hence, are not simply noise).

Finally, a third, and often unrecognized, assumption is that processing is consistent and constant not only over individual participants, but also over the course of the task.
Although studies have been designed to document changes in the pattern of brain activation with practice (e.g., Raichle et al., 1994), these studies are treated as addressing a distinct domain; researchers have been slow to recognize the general implications of the finding that processing changes with practice. In the present study, we not only examined and compared results from standard group analyses with those from individual-differencesbased analyses, but also examined how the pattern of activated areas varies with practice.

In short, in the present article, we extend the usual neuroimaging approach by investigating how practice alters the neural networks used in different tasks by different individuals. Specifically, we focus here on the question of whether different people recruit different brain areas with practice in different tasks. We first will consider group findings, to establish that practice does in fact alter the pattern of brain areas that are recruited during different tasks, and we then will use individual-differences-based multiple regression analyses to document that the set of active brain areas recruited following practice varies for different people. These findings show that the group analyses seriously underestimate the effects of practice in changing the neural bases of how tasks are performed. But more than that, the regression analyses allow us to tie individual differences in brain activation to differences in behavior. Thus, we ask not simply whether the patterns of brain activation following practice vary for different tasks for different people, but also whether those differences predict performance changes in the tasks.

The present study builds on previous experiments conducted in our lab that focused on brain regions for which activation varies with performance across individuals. In the first such study, Kosslyn, Thompson, Kim, Rauch, and Alpert (1996) asked participants to evaluate shape characteristics of visualized letters (e.g., whether specific uppercase letters have curved lines) while their brains were scanned using positron emission tomography (PET). Regions of interest (ROIs) were selected on the basis of those found to be activated during a similar task in previous studies. The mean RT was calculated for each participant and was used as the dependent variable, which was regressed onto the regional cerebral blood flow (rCBF) in the selected ROIs. The results showed that activation in three regions, including the primary visual cortex, predicted about $88 \%$ of the variance in RTs.

In addition, a more recent PET study extended the scope of this individual-differences approach by comparing four different visual imagery tasks and by showing that largely distinct sets of brain regions predicted performance in the different tasks (Kosslyn et al., 2004). The logic of the approach used in Kosslyn et al.'s (2004) study relied upon the distinction between minimally sufficient and rate-limiting processes, which presumably are implemented in different brain regions (Kosslyn \& Plomin, 2001). A rate-limiting process is a performance bottleneck for a particular task, whereas a minimally sufficient process is not. We can use a simple analogy to illustrate 
this distinction. The final score in many video games does not depend on how hard people press the keys, but it does depend on visuomotor coordination processes. In this case, performance is largely unaffected by finger strength, assuming a strength above the minimal threshold required to press a key. On the other hand, performance depends crucially on the efficiency and speed of visuomotor coordination processes. For other tasks, such as opening a jar, the role of these processes is reversed. Now, finger strength corresponds to the rate-limiting process, and visuomotor coordination to the minimally sufficient process. We stress that for any nontrivial task, there is usually more than one rate-limiting process. For instance, as is indicated by the very term itself, visuomotor coordination draws on both visual and motor processes, as well as on various attention and decision processes used to coordinate perception and movement. To extend our analogy, most tasks are like juggling, where performance depends on both finger strength and visuomotor coordination.

We have built on our earlier findings in the present study, now examining the effects of practice on the two different sorts of processes. As in the earlier study, we identified rate-limiting processes via individual-differencesbased analyses. Specifically, we examined in which brain areas changes in activation with practice are tied to changes in behavior with practice. By definition, only changes in rate-limiting processes can underlie such effects. In contrast, areas for which changes in activation with practice are not related to behavior are assumed, by process of elimination, to implement minimally sufficient processes. In the present study, unlike in Kosslyn et al. (2004), we focused on the effects of practice per se-not simply on predicting behavior in individual blocks of trials.

The tasks used by Kosslyn et al. (2004) were explicitly designed so that they would rely on distinct rate-limiting processes. In the functional magnetic resonance imaging (fMRI) study described here, we used three of the visual imagery tasks employed in the PET study by Kosslyn et al. (2004), because these tasks already have some measure of validity. One major advantage of being able to conduct these types of studies with BOLD fMRI is that it allows event-related designs, which permit one easily to eliminate trials associated with an incorrect behavioral response from the analyses, thereby reducing the amount of noise in the results. Although some fMRI studies have reported correlations between brain activation and performance across individuals (e.g., Ng et al., 2001), such studies have not focused on individual-differences analyses of practice effects. Our study is important because we investigated the utility of using the individual-differences approach with fMRI to study changes in processing that are due to practice in high-level cognitive tasks.

\section{METHOD}

\section{Participants}

Twenty-one normal right-handed volunteers ( 12 males, 9 females; mean age, 20.5 years) participated in the study. All the participants had normal or corrected-to-normal vision and had no history of neurological disease. All the participants gave written informed consent for the study according to the protocols approved by the Harvard University and Massachusetts General Hospital Institutional Review Boards. The participants were undergraduate or graduate students (most of them from Harvard University) or professionals from the Boston area. No participant was aware of the purpose of this study until debriefing. Data from 5 of the 21 participants were not included in the analyses, because of uncorrectable motion artifacts or equipment problems. Thus, the analyses reported below were performed on data from the remaining 16 participants.

\section{Materials}

We presented three tasks while brain activation was monitored with fMRI. All tasks shared the same basic stimulus design: a circle containing three radii that divided it into three equal-sized wedges. This Mercedes symbol was oriented in different ways in different stimuli. As is illustrated in Figure 1, the boundary of the circle differed for the three wedges: For one wedge, the boundary was heavy black; for another, it was dashed; and for the third wedge, the boundary was a fine line. (On each trial in all the tasks, the participants were asked to visualize a block letter or number within this stimulus and to compare the portions of the imaged character that fell in the wedge bordered by the heavy black line with the portions of the imaged character that fell in the wedge bordered by the dashed line.) We used 21 alphanumeric characters that appeared in a familiarization phase in which the participants learned the appearance of the characters, as well as script font characters appearing under the trisected circles that were used later to cue the participants to visualize the characters. Eighteen of the characters were test items, which appeared between 4 and 28 times.

The materials were a subset of those used by Kosslyn et al. (2004) and Mast, Ganis, Christie, and Kosslyn (2003). To summarize, for the study phase, we prepared 17 simple uppercase block letters and four numbers. Each character was presented in a circle, subtending ap-
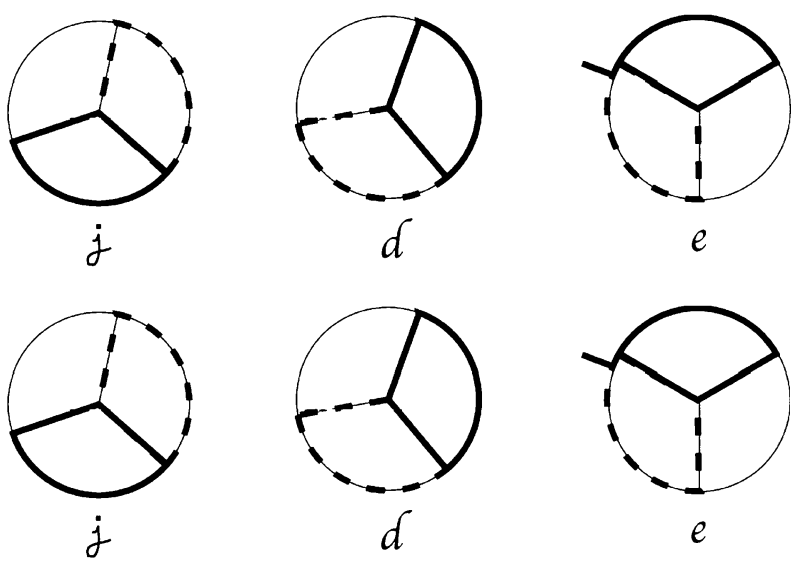

Figure 1. An illustration of sample stimuli for the resolution, inspection, and transformation tasks, respectively. The top line of pictures depicts actual stimuli as presented to the participants for each task; in the bottom line of pictures, the gray character represents the image that the participants would need to form in order to make the judgment appropriate for each task (the answers are bold, dashed, and dashed, respectively). In all the tasks except the inspection task, the participants were asked to judge whether more of the total area of the imaged character would appear within the bold section or the dashed section of the circle. For the inspection task, the participants were asked to judge whether more segments of the imaged character would appear in the bold or the dashed section of the circle. 
proximately $3^{\circ}$ of visual angle. For the test phase, we prepared circles (of the same size as the circles that surrounded each character during the study phase) with a script character beneath each one. The script character was used to cue the participant as to which block character to visualize within the circle.

\section{Procedure}

First, we will summarize the behavioral procedure; then we will review the MRI scanning procedure.

Behavioral procedure. The tasks were administered by a Macintosh G3 Powerbook computer (Apple, Cupertino, CA), using PsyScope software (MacWhinney, Cohen, \& Provost, 1997). The stimuli were projected via a magnetically shielded LCD video projector onto a translucent screen placed behind the head of the participants. The participants could see the stimuli via a front surface mirror mounted on the head coil. Prior to the MRI session, we administered the Edinburgh Handedness Questionnaire (Oldfield, 1971), as well as a general health history questionnaire.

At the beginning of each task, the participants read instructions on the computer screen and then were asked to paraphrase them aloud. Any misconceptions were corrected. Next, they received the stimuli for the study phase. We asked the participants to memorize the appearance of these characters in the following way. A character appeared on the screen within a circle for $5 \mathrm{sec}$ and then disappeared. The participants would then generate a visual mental image of the character in the empty circle. When they felt that they had formed an accurate image, they pressed a button, and the character reappeared. They then compared their mental image with the character, so that they could correct any inaccuracies in their mental representation. This image formation-and-correction procedure was repeated, and then they proceeded to the next character.

Following this, we administered the instructions and 4 practice trials for the first task. The participants were to indicate their judgment by pressing one of two buttons (placed in their dominant hand); both the response and the RT were recorded by the computer. We interviewed the participants immediately after the practice trials to ensure that they understood the task; any misconceptions were corrected, and the participants were asked to paraphrase the instructions again. We asked the participants to make their judgments as quickly as possible, without sacrificing accuracy. MRI scanning began only after the practice trials were complete and it was clear that the participants understood the task. Each MRI session included two functional scans for each task; all three tasks were presented before the second presentation of any task. The order of the tasks was randomized, with the constraint that the order of the second presentation was the same as that of the first presentation. During each scan, we presented 18 trials for each task and used the same stimuli in both blocks for each of the three tasks, but the order of the stimuli was reversed in the second block. On each trial in all the tasks, the participants were asked to visualize a block letter or number within the Mercedes symbol and to compare the portions of imaged character that fell in the wedge bordered by the heavy black line with the portions that fell in the wedge bordered by the dashed line. In addition, the stimuli were designed so that, on half the trials in each task, more of the cued character would be in the dashed boundary wedge and, on half the trials, more of the character would be in the thick black boundary wedge. The trials were arranged so that no more than 3 in succession had more of the character in the wedge with the same boundary. The 4 practice trials for each task had a form identical to that of the test trials, but they included feedback for accuracy. If the participants responded incorrectly, the computer beeped and would not advance to the next trial until the response was corrected. During the experimental trials, there was no such feedback. We employed three tasks, as follows.

For the resolution task, we asked the participants to read the script cue and then to visualize the corresponding block character in the circle, upright, and decide whether more of the character would be in the wedge defined by the heavy black border or in the wedge defined by the dashed line. In the instructions, we provided a diagram that showed the participants how to compare the relative amounts of characters within the divided stimulus circle. Because the discrimination was difficult, the key rate-limiting steps in this task were the processes required to generate images with high resolution and to make fine discriminations while inspecting the image.

For the inspection task, we asked the participants to decide which wedge would have more segments of the visualized character; each segment of a letter corresponds to a stroke typically made when drawing the block character. In the instructions, we provided a diagram that illustrated how to decompose characters into segments and how to count the number of segments in a given wedge. Because the discrimination itself is easy, the critical aspects of this task rely on processes that parse the character into segments and compare the number of segments in each wedge.

For the transformation task, we asked the participants to read the cue, visualize the corresponding block character, and then mentally rotate the character until its top was directly under and aligned with the tick mark. After rotation, the participants were to make the same discrimination as that required in the resolution task. However, in this case, the discrimination was easy; the rate-limiting step in this task lies with processes that rotate the image.

Unlike the PET study by Kosslyn et al. (2004), we used a slow event-related paradigm with 18 trials in each scan. In all cases, the participants were permitted to respond to the trials at their own rate (up to $6 \mathrm{sec}$ poststimulus). However, the intertrial interval was $22.5 \mathrm{sec}$, which was sufficient for the hemodynamic response to return to baseline before the onset of the next trial. A fixation point was present on the screen throughout the intertrial interval. The appropriate stimulus appeared at the onset of each trial; RT measurement started with the appearance of the stimulus on the screen.

MRI procedure. We used a 1.5 T Siemens Allegra scanner. Blood oxygenation changes were monitored by using a T2*-sensitive sequence (gradient echo, TR $=1,500 \mathrm{msec}, \mathrm{TE}=30 \mathrm{msec}$, FOV $=$ $20 \mathrm{~cm}$, flip angle $=90^{\circ}, 64 \times 64$ matrix, voxel size $=3.125 \times$ $3.125 \times 6 \mathrm{~mm}$ ). Each scan resulted in 278 volumes, each composed of fifteen 6-mm axial slices. T1-weighted EPI images acquired at the same locations as the subsequent BOLD images were obtained immediately prior to the functional scans, to facilitate later coregistration of the functional images with the high-resolution structural images. High-resolution, full-volume structural images were collected for all the participants, using SPGR imaging before and after the functional scans (128 1.3-mm-thick sagittal slices, TR = $6.6 \mathrm{msec}, \mathrm{TE}=2.9 \mathrm{msec}, \mathrm{FOV}=25.6 \mathrm{~cm}$, flip angle $=8^{\circ}, 256 \times$ 256 matrix). These T1-weighted images were used for spatial normalization.

MRI statistical analyses. We preprocessed and analyzed the data with AFNI (Cox, 1996), using the following stages: (1) slice timing correction using the AFNI program " $3 \mathrm{dTshift";} \mathrm{(2)} \mathrm{motion}$ correction using the AFNI program "3dvolreg" (Cox \& Jesmanowicz, 1999); (3) spatial smoothing with a Gaussian filter (full-width half-maximum $=4 \mathrm{~mm}$ ), AFNI program " 3 dmerge"; (4) amplitude normalization, by scaling each time series to a mean of 100 and by calculating the percentage of change about this mean; (5) spatial normalization to the MNI305 template (Collins, Neelin, Peters, \& Evans, 1994); and (6) spatial resampling to a $3 \times 3 \times 3 \mathrm{~mm}$ grid. Note that the same spatial transformation was applied to all functional time series. For the functional analyses, the regression model for each condition included four regressors for the noise (thirddegree polynomial) and two regressors for the signal (one for correct and one for incorrect trials). The regressors for the signal were constructed by convolving the task paradigm with a hemodynamic response function with an amplitude equal to 1 (Cohen, 1997). Thus, the signal was composed of all the variance that was accounted for by the experimental stimulus regressors that was not accounted for by the noise regressors. In this slow event-related paradigm, the noise regressors end up modeling mostly the periods of fixation between trials. Only the data for correct trials will be re- 
ported here. The result of this analysis was a map of percentage of signal change for each participant and for each of the six conditions ( 3 tasks $\times 2$ blocks), for a total of 96 maps.

These data were analyzed in two ways. First, we carried out a standard cognitive subtraction fMRI analysis by performing an analysis of variance (ANOVA) on the full data set. The factors used in this ANOVA were task (inspection, resolution, and transformation) and block (first and second). For this analysis, voxel clusters were considered significant if they were composed of at least 5 voxels, all significant at $p<.001$. These parameters provide a good compromise between sensitivity and protection against false positives (Xiong, Gao, Lancaster, \& Fox, 1995).

Next, we performed a linear regression analysis to find clusters in which practice-related activation changes over participants correlated with practice-related changes in the RTs or in the error rates (ERs). Thus, we calculated difference maps for the percentage of signal change maps and difference scores for the behavioral measures (in all cases, the data from the second block were subtracted from the data from the first block). In order to maintain a reasonable power level, we used $p<.005$ and a cluster size of 10 voxels to achieve a familywise alpha of approximately .05 (Xiong et al., 1995). A brain region was considered to be positively correlated with practice-related performance changes if activation changes in the region (e.g., a decrease from Block 1 to Block 2) were associated with changes in the RTs or ERs in the same direction (e.g., a decrease from Block 1 to Block 2). A brain region showing the opposite pattern was considered to be negatively correlated with performance.

For each ROI identified in the regression analysis, we extracted the corresponding mean percentage of signal change value for each participant. Next, we performed separate stepwise forward regression analyses for the RTs and ERs for each task. Note that for each task, only the ROIs that correlated with that particular task went into the stepwise forward regression.

\section{RESULTS}

We first will report the behavioral results and then will turn to the fMRI results.

\section{Behavioral Results}

Table 1 presents the mean RTs and ERs for the three tasks, along with standard errors of the means. The participants were faster for all the tasks during the second block than during the first $[F(1,15)=35.75, p<.0001]$. Least square means comparisons showed that this difference was significant for all three tasks $(p<.001$ in all cases). However, the participants performed some tasks more quickly than others $[F(2,30)=5.79, p<.01]$; specifically, the RTs in the inspection and the resolution tasks did not differ from each other, but both were shorter than those in the transformation task. This pattern of results was the same in both blocks $[F(2,30)=1.07, p>.3$, for the interaction between task and block]. This finding suggests that the participants were not somehow memorizing responses for the individual items; if they had been, the differences among tasks should have been reduced in the second block.

The participants made comparable numbers of errors in the two blocks $[F(1,15)=0.89, p>.3]$. However, they made different numbers of errors in the different tasks $[F(2,30)=5.19, p<.05]$; specifically, the ERs in the resolution and the transformation tasks did not differ from each other, but both were higher than the ERs in the inspection task. Moreover, and of most importance, there was no hint of an interaction between task and block $[F(2,30)=1.22, p>.3]$, again suggesting that the participants did not simply memorize individual stimuli.

We also correlated ER and RT measures for each block for each task (i.e., ERs and RTs for the first block of the inspection task, ERs and RTs for the second block of the inspection task, etc.). There were no significant correlations between ERs and RTs in the same task (all $p \mathrm{~s}>.1$ ), indicating the absence of speed-accuracy tradeoffs. However, we did find significant correlations between the RTs in the first and the second blocks for each task (all $p \mathrm{~s}<.01$, Bonferroni-corrected for multiple comparisons), which suggests that the tasks were reliable.

To ensure that the practice effects were in fact selective and not the result of nonspecific factors (such as increased facility producing responses or comfort with the experimental situation), we calculated the correlation between the practice effects for the three tasks across individuals, for RTs and ERs separately. None of these correlations was significant (all $p \mathrm{~s}>.1$ ), which indicates that the practice effects were not due to a general, nonspecific factor. In addition, it is possible that the practice effects were highly correlated with individual differences in overall performance; that is, the slowest individuals might tend to show the largest practice effects in all the tasks. To examine this possibility, we also calculated the correlation between the practice effects for each task and overall performance (defined for each individual as RTs, or ERs, averaged over tasks and blocks). The practice effects in the three tasks did not correlate with overall performance (all $p \mathrm{~s}>.4$ ), which shows that the practice effects were not due to individual differences in overall performance. Finally, it is possible that the practice effects were highly correlated with individual differences in overall performance on the same task; for instance, the slowest individuals on one of the tasks might show the

Table 1

Mean Response Times (in Milliseconds) and Error Rates (in Percentages) for Each of the Four Tasks in Each of the Two Blocks (With Standard Errors of the Means)

\begin{tabular}{|c|c|c|c|c|c|c|c|c|}
\hline \multirow[b]{3}{*}{ Task } & \multicolumn{4}{|c|}{ Response Times } & \multicolumn{4}{|c|}{ Error Rates } \\
\hline & \multicolumn{2}{|c|}{ Block 1} & \multicolumn{2}{|c|}{ Block 2} & \multicolumn{2}{|c|}{ Block 1} & \multicolumn{2}{|c|}{ Block 2} \\
\hline & $M$ & $S E M$ & $M$ & $S E M$ & $M$ & $S E M$ & $M$ & $S E M$ \\
\hline Inspection & 4,243 & \pm 198 & 3,473 & \pm 168 & 7.3 & \pm 1.6 & 5.1 & \pm 1.6 \\
\hline Resolution & 4,214 & \pm 183 & 3,728 & \pm 165 & 14.1 & \pm 2.4 & 12.1 & \pm 2.5 \\
\hline Transformation & 4,690 & \pm 249 & 4,070 & \pm 182 & 12.9 & \pm 2.6 & 14.4 & \pm 3.0 \\
\hline
\end{tabular}


largest practice effects on that task. To investigate this possibility, we calculated the correlation between practice effects for each task and overall performance on that same task (defined for each task and individual as RTs, or ERs, averaged over blocks). All the correlations were not significant (all $p \mathrm{~s}>.1$ ), which indicates that the practice effects for each task were not an artifact of overall performance on that same task.

\section{MRI Results}

We began by analyzing the data in the traditional way, by looking at the group effects that were consistent across individuals, regardless of performance. Following this, we will report the individual-differences analyses.

Group analyses. All the conditions activated a large set of brain regions in common, when compared with the fixation baseline. Not surprisingly, given the low-level baseline employed, these included large portions of the occipital, parietal, temporal, and frontal lobes, as well as several subcortical areas. Table 2 shows the brain regions for which there was a main effect of task; for these regions, there was more activation during one condition than during another. Because the tasks were specifically designed with the regression approach in mind and because the emphasis is on the effects of practice over tasks, we will not discuss further brain activations that did not change with practice but will focus, instead, on the effects of practice.

Table 3 shows brain regions for which there was a main effect of block in the ANOVA. Many brain regions had less activation in the second block than in the first, including regions in the inferior frontal gyrus (bilaterally), the left cingulate, the right cerebellum, and the right putamen. However, the right anterior cingulate and the right insula, which were deactivated during the first block, had increased activation during the second block. Table 4 shows brain regions for which there was an interaction of task and block - that is, regions for which the effect of block was not uniform across tasks. Planned comparisons revealed that the task $\times$ block interaction was due entirely to the inspection task. In the parahippocampal gyrus regions that showed this interaction, all the activations were negative during the first block. In contrast, during the second block, activation during the resolution and the transformation tasks remained the same, but activation in the inspection task increased.

Individual-differences analyses. The group analyses indicated that practice had different effects in the inspection task than in the other two tasks. However, these results were not dramatic and were evident in only two brain areas. We now ask whether these unimpressive results in fact reflect another, higher order interactionspecifically, with participants. That is, because standard group analyses rely on generalization over participants (which forms the basis of the statistical error term), they will not reveal areas that, in fact, change activation differently for different tasks as they are practiced if this occurs differently for different people.

To address this question, we regressed differences in RT and ER from Block 1 to Block 2 onto differences in activation between the two blocks and looked for brain regions that predicted the behavioral measures. Activation in a total of 15 and 8 regions predicted the RTs and the ERs, respectively. These regions are shown in Table 5. For each task, we entered into a stepwise regression analysis the values of activation in the corresponding regions that predicted behavior. Variations in the changes of RT and ER in all the conditions were predicted by between one and three independent variables entered into the stepwise regressions, with the exception of the ERs in the resolution task, which were not predicted by any variable. The total variance explained for each dependent variable ranged from $76.4 \%$ to $93 \%$.

Tables 5, 6, and 7 list the ROIs associated with each of the dependent variables for RTs and ERs in these analyses. Tables 6 and 7 also list the percentage of variance explained by each variable. We found that variation in the effects of practice on performance in the inspection task was accounted for by five ROIs (three for RTs and two for ERs): the right dorsolateral prefrontal cortex (DLPFC), right Area 18, the left inferior parietal lobule, the left fusiform gyrus, and the left inferior frontal gyrus. Variation in the effects of practice on performance in the resolution task was accounted for by two ROIs (both of

Table 2

Main Effect of Task

\begin{tabular}{|c|c|c|c|c|c|c|}
\hline Area & $x$ & $y$ & $z$ & Tra $>$ Ins & Tra $>$ Res & Ins $>$ Res \\
\hline Left superior parietal lobule (BA 7) & -26 & -53 & 54 & * & * & \\
\hline Right superior parietal lobule (BA 7) & 19 & -58 & 56 & $*$ & $*$ & \\
\hline Right BA 19 & 28 & -81 & 29 & $*$ & $*$ & \\
\hline Right BA 18 & 33 & -89 & 11 & $*$ & $*$ & \\
\hline Left BA 18 & -16 & -95 & 13 & $*$ & $*$ & \\
\hline Right cerebellum & 7 & -50 & -6 & $*$ & $*$ & \\
\hline Left lingual (BA 19) & -13 & -53 & 7 & & $*$ & * \\
\hline Right superior temporal (BA 22) & 52 & -39 & 11 & & $*$ & $*$ \\
\hline Left middle temporal (BA 37) & -45 & -62 & 0 & & $*$ & $*$ \\
\hline Right cerebellum & 14 & -41 & -13 & & $*$ & $*$ \\
\hline Right inferior parietal lobule (BA 40) & 29 & -37 & 48 & & & $*$ \\
\hline
\end{tabular}

Note-The asterisks indicate which contrasts were significant for each region $(p<.01)$. Tra, transformation; Ins, inspection; Res, resolution; BA, Brodmann's area. 
Table 3

Main Effect of Block

\begin{tabular}{lrrrcc}
\hline \multicolumn{1}{c}{ Area } & \multicolumn{1}{c}{$x$} & \multicolumn{1}{c}{$y$} & \multicolumn{1}{c}{$z$} & Block 1 > Block 2 & Block 1 < Block 2 \\
\hline Left inferior frontal (BA 44) & -49 & 8 & 27 & $*$ & $*$ \\
Left inferior frontal (BA 47) & -31 & 19 & -4 & $*$ & $*$ \\
Left cingulate (BA 32) & -6 & 23 & 42 & $*$ & $*$ \\
Right inferior frontal (BA 44) & 46 & 9 & 27 & & $*$ \\
Right cerebellum & 12 & -36 & -12 & & $*$ \\
Right putamen & 23 & 6 & -6 & -3 & \\
Right anterior cingulate (BA 24/32) & 2 & 38 & -3 & \\
Right insula & 34 & -18 & -2 & & $*$ \\
\hline
\end{tabular}

Note-The asterisks indicate which contrasts were significant for each region $(p<.01) . \quad$ BA, Brodmann's area.

them for RTs): the right caudate and the right lingual gyrus. Finally, variation in the effects of practice on performance in the transformation task was accounted for by four ROIs (two for RTs and two for ERs): two left inferior frontal gyrus regions, the right inferior frontal gyrus, and the left cingulate.

To demonstrate the specificity of the brain regions identified in the regression analyses, for each task we took the corresponding ROIs and attempted to predict the performance data for the other two tasks. For instance, we took the areas that predicted the RTs in the resolution task and attempted to predict the RTs for the inspection and the transformation tasks by entering the corresponding brain activation values for those tasks into a multiple linear regression. In all cases, the resulting models accounted for minimal variance and were far from significant (all $p \mathrm{~s}>.2$ ).

To demonstrate further the specificity of each ROI, we compared the correlation coefficients between brain activation in each ROI and performance in all three tasks. For instance, we took an ROI that predicted the RTs in the inspection task and compared directly the correlation coefficient between brain activation and the RTs in the inspection task with those in the remaining two tasks (Steel, Torrie, \& Dickey, 1997). Again, we found for each ROI that brain activation predicted the performance for the task in which it emerged during the analysis significantly better than it did for the other tasks ( $p<.05$ in all cases).

\section{DISCUSSION}

The primary result of this study is that the variations in performance in the different tasks that were due to practice were predicted by variations in activation in different sets of brain regions for different people. Most of these brain regions were different from those found with a standard group ANOVA. We will now discuss the results in more detail.

We will begin by considering the primary issue that motivated this investigation - namely, the nature of practice effects on brain activation. The group ANOVA revealed changes due to practice that were consistent for all three tasks in a number of brain areas (see Table 3). In most such brain regions, there was less activation during the second block than during the first, for all the tasks. However, for two areas, one in the anterior cingulate and one in the right insula, there was actually greater activation during the second block. The regions in which there was greater activation in Block 1 than in Block 2 (including the inferior frontal gyrus bilaterally, the left cingulate, and the cerebellum) may be involved in attention and cognitive control of other brain mechanisms involved in the tasks. From these data, one might suggest that these processes will be more automatic the second time the task is performed - that is, there will be less of a burden on cognitive control processes. These findings and this interpretation are generally consistent with those from other studies of practice effects on brain activation. For instance, the study by Petersen, van Mier, Fiez, \& Raichle (1998) showed a similar general pattern of effects during verb generation and maze-tracing tasks (with the same stimuli retested, as in the present study). In their study, activation decreased in most areas with practice, but in some areas activation actually increased (different ones for the different tasks).

A crucial question is whether comparable effects of practice occur across tasks that are not as different as those used by Petersen et al. (1998). In our study, the task $\times$ block interaction in the ANOVA documented only two areas in the medial temporal lobe (bilaterally, in the vicinity of the parahippocampal gyrus) that showed an effect of practice that varied for the different tasks (see Table 4). In these areas, there was no effect of practice on the resolution and transformation tasks, but there was a strong increase in activation in the inspection task. The inspection task was the only task that required individuals to parse the memorized characters in a novel way (that is, as sets of segments). We speculate that the participants attempted to retrieve the characters as segment sets during the second presentation of the task, after having parsed them as such during the first presentation of

Table 4

Interaction of Task and Block

\begin{tabular}{crrc}
\hline Area & $x$ & $y$ & $z$ \\
\hline Right parahippocampal & 24 & -5 & -18 \\
Left parahippocampal & -24 & -11 & -26 \\
\hline
\end{tabular}

Note-The listed areas showed different effects from Block 1 to Block 2 in different tasks. 
Table 5

Brain Regions in Which Changes in Activation With Practice Were Correlated With Changes in Behavior With Practice (Response Times and Error Rates) in the Three Tasks

\begin{tabular}{|c|c|c|c|c|c|c|c|}
\hline Measure & Task & Area & $x$ & $y$ & $z$ & $r$ & $p$ \\
\hline \multirow[t]{15}{*}{ Response time } & \multirow[t]{8}{*}{ Inspection } & *Right dorsolateral prefrontal cortex (BA 10/46) & 28 & 35 & 22 & -.85 & $<.0001$ \\
\hline & & *Left inferior parietal lobule (BA 40) & -57 & -35 & 31 & -.78 & $<.001$ \\
\hline & & *Right BA 18 & 16 & -100 & 11 & .76 & $<.001$ \\
\hline & & Left inferior frontal (BA 44/45) & -57 & 21 & 11 & -.83 & $<.0001$ \\
\hline & & Right caudate/putamen & 22 & 19 & 7 & -.83 & $<.0001$ \\
\hline & & Left middle temporal (BA 21) & -56 & -4 & -9 & -.82 & $<.0001$ \\
\hline & & Left claustrum & -29 & 6 & 4 & -.79 & $<.001$ \\
\hline & & Right putamen & 22 & -2 & 16 & -.76 & $<.001$ \\
\hline & \multirow[t]{2}{*}{ Resolution } & *Right lingual (BA 19) & 15 & -54 & 6 & .78 & $<.001$ \\
\hline & & *Right caudate & 1 & 13 & 10 & -.79 & $<.001$ \\
\hline & \multirow[t]{5}{*}{ Transformation } & ${ }^{*}$ Left inferior frontal (BA 44) & -49 & 9 & 23 & -.83 & $<.001$ \\
\hline & & *Left inferior frontal (BA 47) & -35 & 27 & -6 & .86 & $<.0001$ \\
\hline & & Left middle temporal (BA 21) & -48 & 2 & -22 & .79 & $<.001$ \\
\hline & & Right anterior cingulate (BA 24/32) & 12 & 27 & 21 & .80 & $<.001$ \\
\hline & & Right inferior frontal (BA 47) & 24 & 18 & -15 & .89 & $<.0001$ \\
\hline \multirow[t]{8}{*}{ Error rate } & \multirow[t]{4}{*}{ Inspection } & *Left fusiform (BA 37) & -29 & -58 & -10 & .77 & $<.001$ \\
\hline & & *Left inferior frontal (BA 47) & -38 & 11 & -7 & .82 & $<.0001$ \\
\hline & & Right inferior parietal lobule (BA 40) & 40 & -34 & 42 & .79 & $<.001$ \\
\hline & & Right cerebellum & 29 & -33 & -18 & .80 & $<.001$ \\
\hline & \multirow[t]{4}{*}{ Transformation } & *Left cingulate (BA 32) & -6 & 34 & 29 & -.85 & $<.0001$ \\
\hline & & *Right inferior frontal (BA 44) & 17 & 54 & 25 & -.81 & $<.001$ \\
\hline & & Left superior temporal (BA 22) & -45 & -48 & 21 & -.82 & $<.0001$ \\
\hline & & Right superior temporal (BA 22) & 51 & -53 & 12 & -.81 & $<.001$ \\
\hline
\end{tabular}

Note-Areas in which changes in activation predicted behavior in the stepwise regressions (as opposed to the first-order correlation) are preceded by an asterisk. BA, Brodmann's area.

the task (cf. Rombouts, Barkhof, Witter, Machielsen, \& Scheltens, 2001).

By definition, the task $\times$ block interaction identifies brain regions in which practice had a different effect for different tasks, but in a consistent way across participants. However, the ANOVA that produced this interaction is likely to overlook areas for which the effect of practice across tasks is not consistent across participants. Indeed, the regression analyses of practice effects revealed that brain activation in several regions was af- fected by practice in different ways, depending on the task (see Tables 6 and 7). To our knowledge, this is the first study to show that practice affects different people's brains in different ways for different tasks. The areas that best predicted the effects of practice on RTs across individuals in the inspection task were the right DLPFC, right BA18, and the left inferior parietal lobule. The areas that best predicted the effects of practice on ER across individuals were the left fusiform gyrus and the left inferior frontal gyrus. The frontal and parietal areas

Table 6

Results of the Stepwise Forward Multiple Regression Analyses on the Effect of Practice for Response Times (RTs)

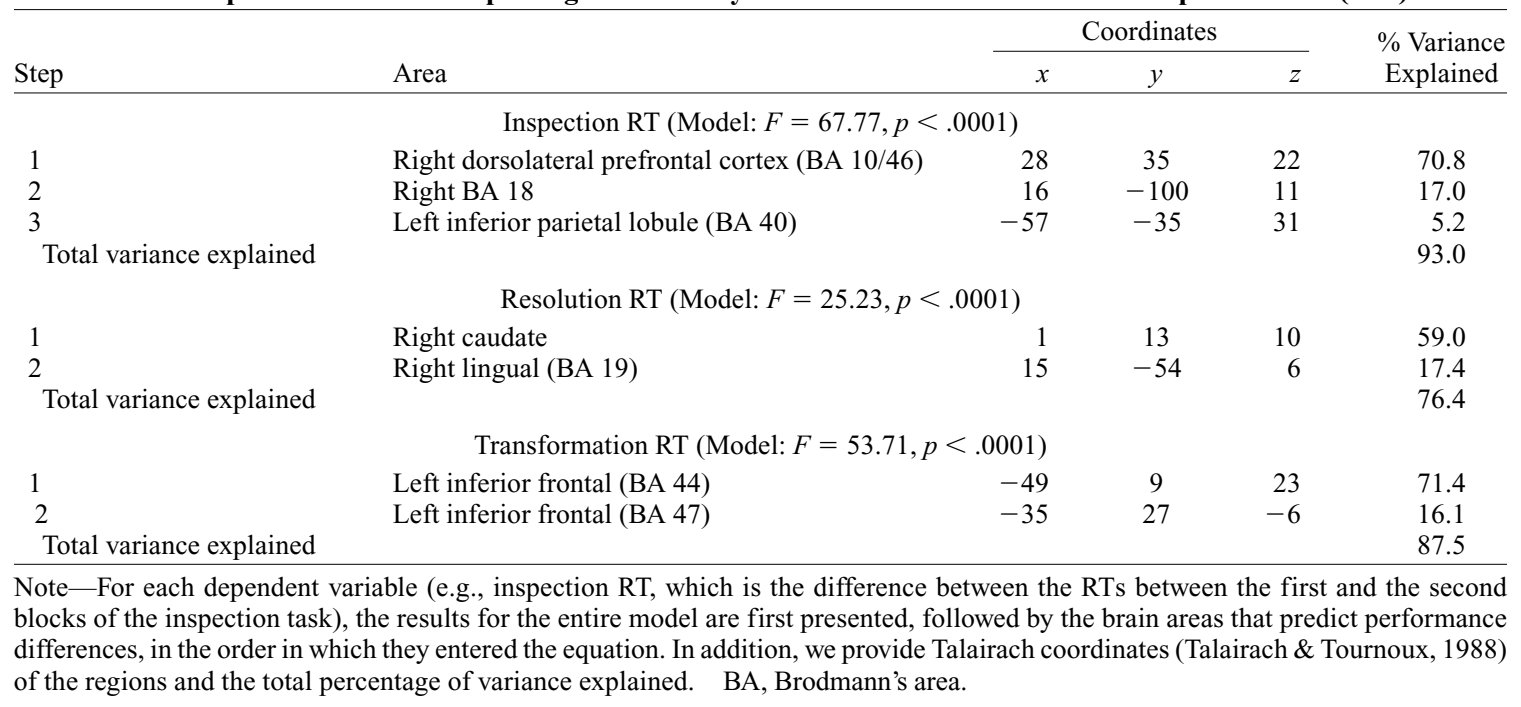


Table 7

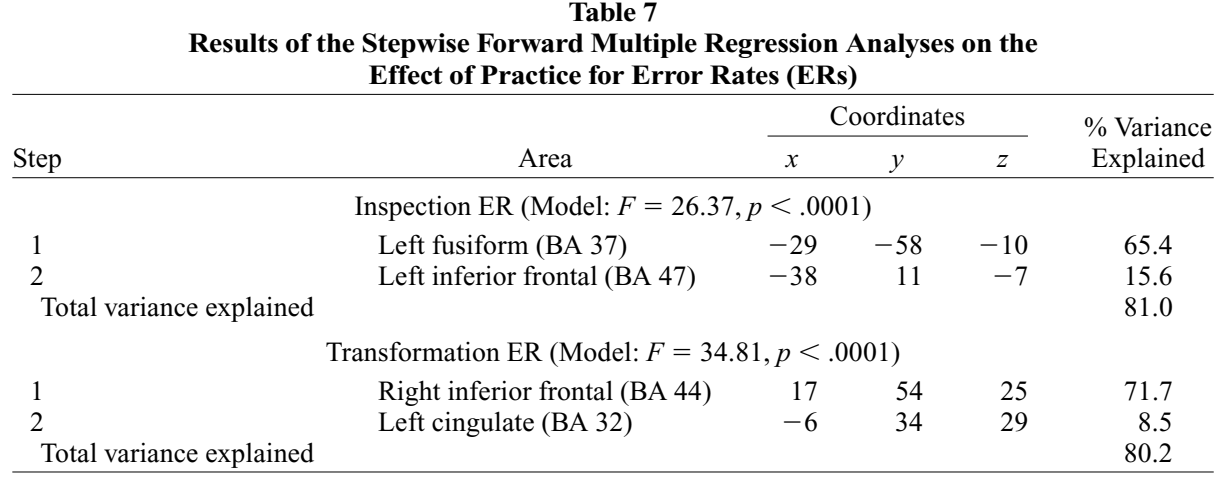

Note-For each dependent variable (e.g., inspection ER, which is the difference between the error rate between the first and second blocks of the inspection task), the results for the entire model are first presented, followed by the brain areas that predict performance, in the order in which they entered the equation. Note that no areas predicted the ER in the resolution task. In addition, we provide Talairach coordinates (Talairach \& Tournoux, 1988) of the regions and the total percentage of variance explained. BA, Brodmann's area.

were negatively correlated with changes in performance, whereas the occipital and temporal areas were positively correlated with changes in performance. For areas that are positively correlated with practice effects, the less activation there is in the second block (relative to the first block), the larger the advantage in performance as a result of practice. These areas may implement processes used during the initial phases of performing novel tasks, but these processes are used less as performance improves with practice; participants who continue using these areas in the second block tend to show smaller performance improvements as a result of practice. In contrast, for areas that are negatively correlated with practice effects, the more activation there is in the second block (relative to the first), the larger the advantage in performance as a result of practice. These areas may implement processes that become more important after the task is no longer novel; participants who do not begin using these processes in the second block tend to show smaller performance gains as a result of practice.

For the inspection task, one could hypothesize that initial performance on this task is driven by the visual areas (the left fusiform gyrus, right Area 18) that show a positive correlation with practice effects. With practice, perhaps after participants learn how to organize the segments that make up the characters, processes implemented in the fronto-parietal areas (left inferior parietal, left inferior frontal, and the right DLPFC) may take on the most important role.

In contrast, the areas that best predicted the effects of practice on RTs across individuals in the resolution task were the right caudate and the right lingual gyrus. Changes in activation in the caudate were negatively correlated with practice, whereas changes in the lingual gyrus were positively correlated. These findings might suggest that visual areas are involved in the task at the outset but that the caudate becomes more important with practice, perhaps because participants learn how to make the judgments more efficiently.
Finally, the areas that best predicted the effects of practice on RTs over individuals in the transformation task were two foci in the left inferior frontal gyrus (BAs 44 and 47), and the areas that best predicted the effects of practice on ER across individuals were the right inferior frontal gyrus (BA 44) and the left cingulate. The only area that was positively correlated with practice was in the left inferior frontal gyrus (BA 47). We note that the center of mass of two of the clusters found in the analysis of the data from this task were within a few millimeters from the center of mass of two areas that showed a main effect of repetition in the group ANOVA (see Table 3). Both of these clusters were in the left inferior frontal gyrus (BAs 44 and 47). These regions were strongly correlated with performance changes due to practice, but with the opposite sign. This is a very important observation, because one would conclude, on the basis of the ANOVA, that these regions become less important with practice, because on average they become less active with practice. The individualdifferences analysis presented here, in contrast, reveals that these two areas behave in opposite ways, even though, on average, activation gets smaller with practice: The left BA 44 appears to become more important with practice, because participants who show the largest practice gains in RTs also have the largest relative increase in brain activation, whereas the left BA 47, with its positive correlation, appears to fade out with practice in terms of its importance to task performance. Figure 2 illustrates this pattern of results by showing how changes in RT varied along with changes in brain activation in voxels that overlapped in the two analyses (independently for each of the two left inferior frontal regions). Figure 2 indicates also that the effects were specific for the transformation task, showing that there was virtually no correlation in these regions between RTs and brain activation for the other two tasks. This pattern may partly reflect the use of different strategies to perform the transformation task in the two blocks, which may rely on different cognitive control processes. This case clearly illustrates how the group and the 
Left Inferior Frontal (BA 44)
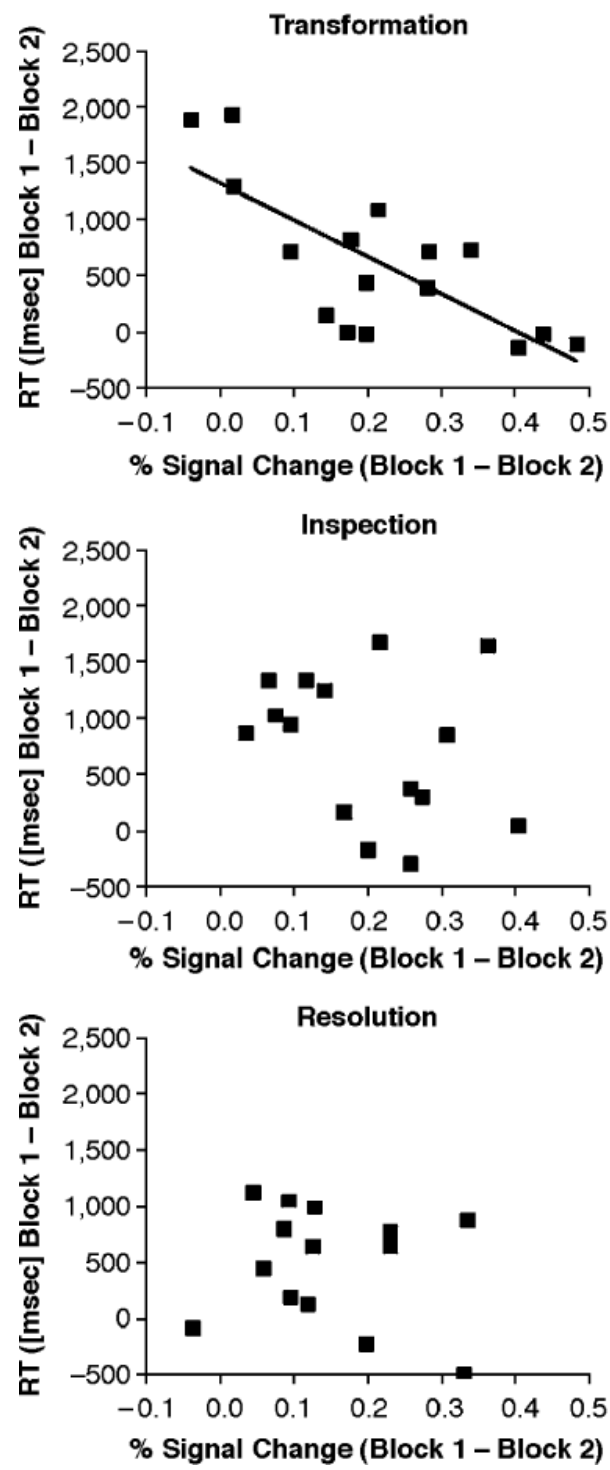

Left Inferior Frontal (BA 47)

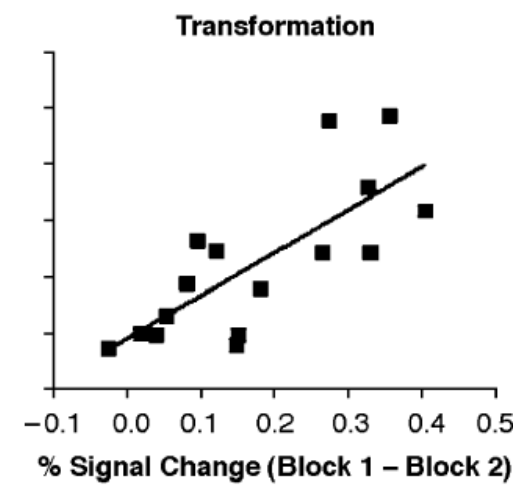

Inspection

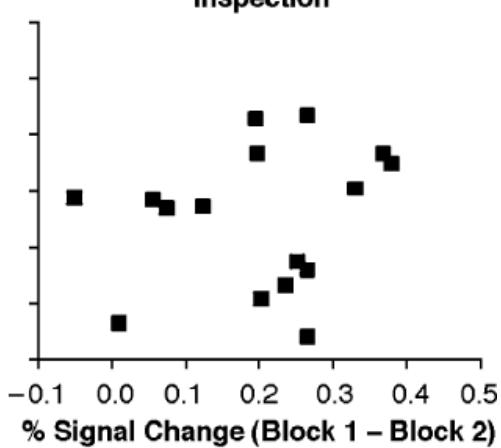

Resolution

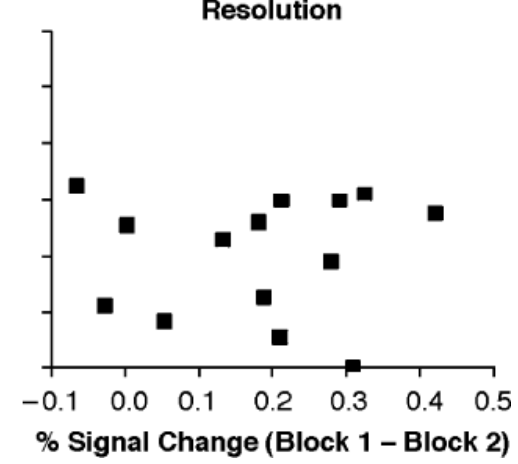

Figure 2. An illustration of the pattern of effects in left inferior frontal cortical areas BA 44 and BA 47. The ANOVA revealed an overall decrease in activation in Block 2, in comparison with Block 1, in these two regions. Furthermore, individual differences in (changes in) activation in these areas predicted the (changes in) response times (RTs) in the transformation task, but in different ways: The correlation in BA 44 was negative, whereas that in BA 47 was positive. The scatterplots illustrate the practice effect in RTs ( $y$-axis) against the practice effects in regional cerebral blood flow $(x$-axis). The top, middle, and bottom panels show the results for the transformation, inspection, and resolution tasks, respectively. Note that variations in activation in these regions do not predict performance in the inspection and resolution tasks, which is why least squares regression lines were plotted only for the transformation condition.

individual-differences analyses can be complementary. It also illustrates the potential importance of considering individual-differences data when interpreting brainimaging findings. Almost by definition, the individualdifferences approach is most useful in the study of highlevel cognitive phenomena that are characterized by large individual variability, such as those in this study. However, this approach may be useful even in the study of low-level cognitive phenomena that are usually characterized by low individual variability, if the tasks are made sufficiently challenging to increase such individual variability.

One could argue that our study does not go beyond standard subtraction methods, because after all, we still used a fixation baseline to calculate the percentage of 
signal changes. However, with BOLD fMRI, it is always necessary to measure activation relative to something else during the same scan. In this study, we used a lowlevel baseline (fixation between trials) to try to remove as little activation as possible. In accord with the idea that a fixation baseline elicits consistent activation by itself (e.g., Gusnard \& Raichle, 2001), we did find consistent deactivation in a number of regions during all the tasks, relative to baseline. These regions included parts of the medial frontal cortex, parts of the medial temporal lobe, the retrosplenial cortex, and the posterior parietal lobe. Crucially, this fixation baseline was identical for all the conditions, making it difficult to explain the observed task differences in this way.

In summary, the present study was designed to allow us to answer three general questions. First, are the results of subtraction the same as those obtained with another method, where variations in activation are used to predict variations in performance of the task? With respect to the effects of practice, the answer was clearly no. Groupbased analyses produced very different results from regression-based individual-differences analyses. Second, are all important brain areas consistently activated across participants? Again, the answer was clearly no. Most areas that predicted the effects of practice on performance were not activated consistently by the participants. Finally, does practice affect different areas in different ways for different people in different tasks? The answer was again clear: yes. The precise areas that predicted changes in performance with practice varied for the different tasks, but this was more dramatically and clearly revealed by the individual-differences analyses.

In conclusion, this study is a modest step toward demonstrating how individual-differences analyses may be helpful in revealing brain areas that are overlooked in standard group analyses. This approach is particularly important for interpreting higher order effects, such as the effects of practice studied here. The individual-differences approach and the standard group analysis approach should be used in conjunction, because they reveal complementary facets of the information processing that gives rise to brain activation.

\section{REFERENCES}

Ackerman, P. L., Kyllonen, P. C., \& Roberts, R. D. (Eds.) (1999). Learning and individual differences: Process, trait, and content determinants. Washington, DC: American Psychological Association.

Cohen, M. S. (1997). Parametric analysis of fMRI data using linear systems methods. NeuroImage, 6, 93-103.

Collins, D. L., Neelin, P., Peters, T. M., \& Evans, A. C. (1994). Automatic 3D intersubject registration of MR volumetric data in standardized Talairach space. Journal of Computer Assisted Tomography, 18, 192-205.

Cox, R. W. (1996). AFNI: Software for analysis and visualization of functional magnetic resonance neuroimages. Computers \& Biomedical Research, 29, 162-173.

Cox, R. W., \& JESMANOWICZ, A. (1999). Real-time 3D image registration for functional MRI. Magnetic Resonance in Medicine, 42, 1014-1018. DonDERs, F. (1969). On the speed of mental processes (W. G. Koster, Trans.). In K. Koster (Ed.), Attention and performance II (pp. 412431). Amsterdam: North-Holland. (Original work published 1868)
Ganis, G., Thompson, W. L., \& Kosslyn, S. M. (2004). Brain areas underlying visual mental imagery and visual perception: An fMRI study. Cognitive Brain Research, 20, 226-241.

Ganis, G., Thompson, W. L., Mast, F. W., \& Kosslyn, S. M. (2003). Visual imagery in cerebral visual dysfunction. Neurologic Clinics, 21, 631-646.

Gusnard, D. A., \& Raichle, M. E. (2001). Searching for a baseline: Functional imaging and the resting human brain. Nature Reviews Neuroscience, 2, 685-694.

KANDEL, E. R., \& SQuiRe, L. R. (2000). Neuroscience: Breaking down scientific barriers to the study of brain and mind. Science, 290, 11131120.

Kosslyn, S. M., \& Plomin, R. (2001). Toward a neurocognitive genetics: Goals and issues. In D. D. Dougherty \& S. L. Rauch (Eds.), Psychiatric neuroimaging research: Contemporary strategies (pp. 383402). Washington, DC: American Psychiatric Press.

Kosslyn, S. M., Thompson, W. L., \& Alpert, N. M. (1997). Neural systems shared by visual imagery and visual perception: A positron emission tomography study. NeuroImage, 6, 320-334.

Kosslyn, S. M., ThOMPSON, W. L., Kim, I. J., Rauch, S. L., \& AlPerT, N. M. (1996). Individual differences in cerebral blood flow in area 17 predict the time to evaluate visualized letters. Journal of Cognitive Neuroscience, $\mathbf{8}, 78-82$.

Kosslyn, S. M., Thompson, W. L., Shephard, J. M., Ganis, G., Bell, D., Danovitch, J., Wittenberg, L. A., \& Alpert, N. M. (2004). Brain $\mathrm{rCBF}$ and performance in visual imagery tasks: Common and distinct processes. European Journal of Cognitive Psychology, 16, 696716.

MacWhinney, B., Cohen, J., \& Provost, J. (1997). The PsyScope experiment-building system. Spatial Vision, 11, 99-101.

Mast, F. W., Ganis, G., Christie, S., \& Kosslyn, S. M. (2003). Four types of visual mental imagery processing in upright and tilted observers. Cognitive Brain Research, 17, 238-247.

NG, V. W. K., Bullmore, E. T., de Zubicaray, G. I., Cooper, A., SucKLING, J., \& Williams, S. C. R. (2001). Identifying rate-limiting nodes in large-scale cortical networks for visuospatial processing: An illustration using fMRI. Journal of Cognitive Neuroscience, 13, 537545 .

OLDFIELD, R. C. (1971). The assessment and analysis of handedness: The Edinburgh inventory. Neuropsychologia, 9, 97-113.

Petersen, S. E., van Mier, H., Fiez, J. A., \& Raichle, M. E. (1998), The effects of practice on the functional anatomy of task performance. Proceedings of the National Academy of Sciences, 95, 853-860.

Posner, M. (1978). Chronometric explorations of mind. Hillsdale, NJ: Erlbaum.

Raichle, M. E., Fiez, J. A., Videen, T. O., Macleod, A. M., Pardo, J. V., Fox, P. T., \& Petersen, S. E. (1994). Practice-related changes in human brain functional anatomy during nonmotor learning. Cerebral Cortex, 4, 8-26.

Rombouts, S. A., Barkhof, F., Witter, M. P., Machielsen, W. C., \& SCHELTENS, P. (2001). Anterior medial temporal lobe activation during attempted retrieval of encoded visuospatial scenes: An eventrelated fMRI study. NeuroImage, 14, 67-76.

SARTORI, G., \& Umiltà, C. (2000). How to avoid the fallacies of cognitive subtraction in brain imaging. Brain \& Language, 74, 191-212.

Smith, E. E., \& Jonides, J. (1997). Working memory: A view from neuroimaging. Cognitive Psychology, 33, 5-42.

Steel, R. G. D., Torrie, J. H., \& Dickey, D. A. (1997). Principles and procedures of statistics: A biomedical approach (3rd ed.). New York: McGraw-Hill.

STERNBERG, S. (1969). The discovery of processing stages: Extensions of Donders' method. Acta Psychologica, 30, 276-315.

TAlairach, J., \& Tournoux, P. (1988). Co-planar stereotaxic atlas of the human brain: 3-dimensional proportional system. An approach to cerebral imaging (M. Rayport, Trans.). New York: Thieme.

Xiong, J., GaO, J.-H., Lancaster, J. L., \& Fox, P. T. (1995). Clustered pixel analysis for functional MRI activation studies of the human brain. Human Brain Mapping, 3, 287-301.

(Manuscript received September 26, 2004; revision accepted for publication April 6, 2005.) 